Accepted ver.

Expert systems with applications. - ISSN 0957-4174. - Vol. 42, no. 4 (2015):2198-2204 doi: 10.1016/j.eswa.2014.09.060

\title{
Comparison of Some Aggregation Techniques Using Group Analytic Hierarchy Process
}

\author{
Petra Grošelj ${ }^{1}$, Lidija Zadnik Stirn ${ }^{1}$, Nadir Ayrilmis², Manja Kitek $\operatorname{Kuzman}^{1}(凹)$ \\ ${ }^{1}$ University of Ljubljana, Biotechnical Faculty Jamnikarjeva 101, 1000 Ljubljana, Slovenia \\ ${ }^{2}$ Istanbul University, Forestry Faculty, Bahcekoy 34473, Sariyer, Istanbul, Turkey \\ E-mail: petra.groselj@bf.uni-lj.si, lidija.zadnik@bf.uni-lj.si,nadiray@istanbul.edu.t \\ manja.kuzman@bf.uni-lj.si
}

(凶) Corresponding author Tel: +386 13203 604, Fax: +386 12572 297,

\begin{abstract}
Group decision making is an important part of multiple criteria decision making and the analytic hierarchy process (AHP). The aim of this paper was to compare group AHP methods. Seven simple group AHP aggregation techniques that could be attractive for applications selected from the vast array of group AHP models proposed in the literature were selected for evaluation. We developed three new measures of evaluation: group Euclidean distance, group minimum violations, and distance between weights for the purpose of evaluation. The results of seven group AHP methods of the theoretical example were evaluated by three new evaluation measures, satisfactory index and fitting performance index. Furthermore, a case study of a decision making problem from the construction engineering field was performed and nine group AHP aggregation techniques, seven of them formerly presented and two new two stage group approaches were applied. Finally, the case study was evaluated using all five measures for each of the nine group decision making methods. The results showed that not all group AHP methods are equally convenient and that the selection of the method depended on the specific application.
\end{abstract}

Keywords: Decision analysis; Decision support systems; Analytic hierarchy process; Group decision making, Construction; wood 


\section{Introduction}

Group decision making is becoming an increasingly important part of multiple criteria decision making (Ishizaka \& Labib, 2011a; Ahmad et al., 2014; Kuzman et al., 2013; Skorupski, 2014; Ren et al., 2013; Yu and Lai, 2011; De Brucker et al., 2013; Wang et al., 2014). Multiple stakeholders can contribute a variety of experiences, expertise and perspectives, and a group can better deal with the complexity of the problem than a single decision maker (DM). The analytic hierarchy process (AHP) (Saaty, 1980) is deemed to be one of the most appropriate methods for group multiple criteria decision making (Peniwati, 2007). In group AHP, four basic approaches for deriving the group priority vector from comparison matrices of DMs are suggested (Dyer and Forman, 1992; Lai et al., 2002; Ishizaka \& Labib, 2011b). The group can try to reach a consensus on a meeting, first in developing the hierarchy and then in generating pairwise comparisons. If they cannot reach a consensus regarding a particular judgment, they can vote or try to achieve a compromise. Social choice theory with voting systems (Taylor and Pacelli, 2008) can be combined with AHP (Srdjevic, 2007). The aggregation of individual priorities (AIP) and the aggregation of individual judgments (AIJ) are two main mathematical aggregating methods (Forman and Peniwati, 1998). The most widely used aggregation technique is the weighted geometric mean method for AIJ (WGM-AIJ), which has been applied in numerous applications (Sun \& Li, 2009; Ananda \& Herath, 2008; Cortés-Aldana et al., 2009; Lee et al., 2009; Srdjevic et al., 2013, de Luca, 2014).

The decision maker is satisfied if the final group priorities are as close as possible to his judgments, priorities or his ranking of criteria. Unlike the single DM case, in the group case there are not many studies comparing the results of different AHP group approaches, which 
results in a lack of measures for comparing group methods (Huang, 2009; Hosseinian et al., 2012).

The main objective of this paper is to develop new measures for evaluating AHP group methods. We proposed three new measures: group Euclidean distance (GED), group minimum violations (GMV), and distance between weights (WD). The second aim of the present study was to select the most appropriate group AHP method for employment in the applications. Although WGM-AIJ is the most often applied method it is not necessary the most suitable method. For the comparative study we selected WGM-AIP, weighted arithmetic mean method (WAM), and some recently presented models in addition to WGM-AIJ (Regan et al., 2006; Sun \& Greenberg, 2006; Huang, 2009; Hosseinian et al., 2012). These models were selected because they are easy to understand and could be attractive for many applications.

The three new measures, the satisfactory (SAT) index (Huang, 2009) and the fitting performance (FP) index (Hosseinian et al., 2012) were employed in the evaluation study, which compared seven group AHP methods in a theoretical example. Additionally, a case study that compared the criteria for selecting building construction method and material for an industrial type of building was performed. In the study, three groups of stakeholders were included in the decision making. To aggregate the stakeholders' judgments we suggest utilizing AIJ within the groups first and then AIP between the groups. In the paper we proposed two new stage group approaches, namely WGM-WAM and WGM-LW-AHP. Seven known group AHP methods and two newly proposed were applied in the case study for deriving group priorities. The results of nine group AHP methods were compared with five evaluation measures: GED, GMV, WD, SAT index, and FP index. 
The next section offered a brief description of group AHP methods applied in the study. Further, we proposed the measures for evaluating the group AHP methods. The theoretical part of paper was followed by the theoretical example and a case study. Finally, some conclusions were provided.

\section{Revision of group AHP prioritization methods}

Let $n$ be the number of criteria (or alternatives) and $m$ the number of DMs. The standard AHP 1-9 scale (Saaty, 1980) was used for the judgments of each DM, which were written in the comparison matrices $\mathbf{A}^{(k)}=\left(a_{i j}^{k}\right)_{n \times n}, k=1, \ldots, m$. If the DMs' opinions were not equally important, the relative importance weight of $k$-th DM's opinion was denoted by $\alpha_{k}$, for $k=1, \ldots, m$, with $\alpha_{k}>0$ and $\sum_{k=1}^{m} \alpha_{k}=1$

There are many methods for deriving priority vectors but in this study we primarily used the eigenvector method (Saaty, 1980) resulting in $\mathbf{w}^{k}=\left(w_{1}{ }^{k}, \ldots, w_{n}{ }^{k}\right)^{T}, k=1, \ldots, m$ as DMs' priority vectors. In the study we focused on the additive error structure $a_{i j}=\frac{w_{i}}{w_{j}}+\varepsilon_{i j}$ for inconsistent comparison matrix $A$ and used additive normalization condition $\sum_{i=1}^{n} w_{i}=1$ for all priority vectors for one or more DMs (Sun \& Greenberg, 2006). The consistency of judgments in the comparison matrix $A$ was measured by the consistency ratio $C R_{A}=\frac{\lambda_{A, \max }-n}{(n-1) R I_{n}}$, where $R I_{n}$ was the average random consistency index. A consistency ratio of less than 0.1 was considered acceptable. 
Of the AIJ methods WGM-AIJ is the only method that meets several required axiomatic conditions, such as the reciprocal property (Aczél \& Alsina, 1986). The individual judgments $a_{i j}^{k}, k=1, \ldots, m$ were aggregated into a group judgment $a_{i j}^{W G M M}$ by weighted geometric mean:

$$
a_{i j}{ }^{W G M M}=\prod_{k=1}^{m}\left(a_{i j}^{k}\right)^{\alpha_{k}}
$$

The group priority vector was derived from the group comparison matrix $A^{\text {WGMM }}$ by the eigenvector method.

The AIP is a suitable method when a group is non-homogenous and consists of stakeholders from different fields. Both the WAM and WGM approaches can be used for the AIP. First, each DM $k, k=1, \ldots, m$, applies for the eigenvector method for deriving the priority vector $\mathbf{w}^{k}=\left(w_{1}{ }^{k}, \ldots, w_{n}{ }^{k}\right)^{T}$ from its comparison matrix. The individual priority vectors are then synthesized into the group priority vector $\mathbf{w}=\left(w_{1}, \ldots, w_{n}\right)^{T}$ using the weighted arithmetic mean (WAM) (2) or weighted geometric mean (WGM-AIP) (3):

$$
\begin{aligned}
& w_{i}=\sum_{k=1}^{m} \alpha_{k} w_{i}^{k}, i=1, \ldots, n ; \\
& w_{i}=\prod_{k=1}^{m}\left(w_{i}{ }^{k}\right)^{\alpha_{k}}, i=1, \ldots, n .
\end{aligned}
$$

\subsection{LW-AHP model}

The Lehrer - Wagner (LW) model (Lehrer \& Wagner, 1981) was adopted for the AHP by Regan et al. (2006). In this study, it was assigned as the LW-AHP model. The base of this model was placed in the philosophy of negotiation (Regan et al., 2006) and used for the AIP. The initial priority vectors ${ }^{0} \mathbf{w}^{k}=\left({ }^{0} w_{1}{ }^{k}, \ldots,{ }^{0} w_{n}{ }^{k}\right)^{T} k=1, \ldots, m$ were derived by the eigenvector 
method from DMs' comparison matrices. They were revised according to weights of respect, $w_{s}^{i j}$, which were based on the strength of the differences between the priorities of DMs for each criterion (or alternative) $s, s=1, \ldots, n$.

$$
w_{s}^{i j}=\frac{1-\left|{ }^{0} w_{s}^{i}-{ }^{0} w_{s}^{j}\right|}{\sum_{j=1}^{m}\left(1-\left|{ }^{0} w_{s}^{i}-{ }^{0} w_{s}^{j}\right|\right)},
$$

The weights are gathered in the matrices of weights of respect $W_{s}=\left(w_{s}^{i j}\right)_{m \times m}$. Let ${ }^{0} P_{s}$ denote the vector of DMs' priorities of the criterion $s:{ }^{0} P_{s}=\left({ }^{0} w_{s}^{1}, \ldots,{ }^{0} w_{s}^{m}\right)^{T}$. The updated priorities of the criterion $s$ after the first round of the aggregation result in ${ }^{1} P_{s}=W_{s}{ }^{0} P_{s}=\left({ }^{1} w_{s}^{1}, \ldots,{ }^{1} w_{s}^{m}\right)^{T}$. The process of aggregation was repeated with the same weights of respect: ${ }^{r} P_{s}=\left(W_{s}\right)^{r}{ }^{0} P_{s}$. As $r$ approaches infinity, the revised priorities of criterion $s$ converged towards the final priority ${ }^{c} w_{s}={ }^{c} w_{s}^{1}=\ldots={ }^{c} w_{s}^{m}$, which was equal for all DMs and where $c$ was the number of iterations needed to reach the convergence.

\subsection{GWLS model}

Sun and Greenberg (2006) proposed a GWLS model for deriving group priorities:

$$
\min \sum_{k=1}^{m} \sum_{j=1}^{n} \sum_{i=1}^{n} \alpha_{k}\left(a_{i j}^{k} w_{j}-w_{i}\right)^{2}
$$

subject to: $\sum_{i=1}^{n} w_{i}=1$,

$$
w_{i}>0, i=1, \ldots n
$$

and proved that the solution of model (5) is given by

$$
w=C^{-1} \hat{\lambda},
$$


where

$$
\begin{aligned}
& C=\tilde{A}+\tilde{A}^{T}-\Lambda, \tilde{A}=\left(\tilde{a}_{i j}\right)_{n \times n}, \tilde{a}_{i j}=\sum_{k=1}^{m} \alpha_{k} a_{i j}{ }^{(k)}, \\
& \hat{a}_{i j}=\sum_{k=1}^{m} \alpha_{k}\left(\left(a_{i j}{ }^{(k)}\right)^{2}+1\right), \eta_{j}=\sum_{i=1}^{n} \hat{a}_{i j}, \Lambda=\operatorname{diag}\left(\eta_{1}, \eta_{2}, \ldots, \eta_{n}\right) \\
& \text { and } C^{-1}=\left(\bar{c}_{i j}\right)_{n \times n}, \hat{\lambda}=\left(\frac{\lambda}{2}, \frac{\lambda}{2}, \ldots, \frac{\lambda}{2}\right)^{T}, \lambda=2 /\left(\sum_{i=1}^{n} \sum_{j=1}^{n} \bar{c}_{i j}\right) .
\end{aligned}
$$

\subsection{PD\&R model}

Huang et al. (2009) proposed a group AHP model considering the differences of preference among criteria (or alternatives) and the ranks of the criteria (or alternatives) for each DM. The priority vector of DM $k, k=1, \ldots, m$ was originally derived by the logarithmic least squares method (Crawford \& Williams, 1985), but we used the eigenvector method instead. The preferential difference between criterion $i$ and criterion $j$ of DM $k$ is defined as:

$$
\theta_{i j}^{k}=\left|w_{i}^{k}-w_{j}^{k}\right|
$$

The preferential differences are used for the AIJ which are gathered in $A^{\text {diff }}=\left(a_{i j}^{\text {diff }}\right)_{n \times n}$ :

$$
a_{i j}^{d i f f}=\left(\prod_{k=1}^{m}\left(a_{i j}^{k}\right)^{\theta_{i j}^{k}}\right)^{\frac{1}{m} \sum_{k=1}^{m} \theta_{i j}^{k}} \text { for } \mathrm{i}, \mathrm{j}=1, \ldots, \mathrm{n}
$$

The eigenvector method is employed for deriving the priority vector $\mathbf{w}^{\text {diff }}$ from $A^{\text {diff }}$. The vector of adjusting weights $\mathbf{w}^{\text {rank }}$, considering the preferential ranks, is defined as

$$
w_{i}^{\text {rank }}=\frac{\delta_{i}}{\sum_{i=1}^{n} \delta_{i}}
$$


where $\delta_{i}$ denotes the sum of rank-adjusting factors for all DMs for the criterion $i$, $\delta_{i}=\sum_{k=1}^{m} \delta_{i}^{k}=\sum_{k=1}^{m} \frac{n}{r_{i}^{k}}$, and $r_{i}^{k}$ the preferential rank of the criterion $i$ according to DM $k$.

The final group priorities of the PD\&R model are the normalized products of $w_{i}^{\text {diff }}$ and $w_{i}^{\text {rank }}$ :

$$
w_{i}=\frac{w_{i}^{\text {diff }} w_{i}^{\text {rank }}}{\sum_{i=1}^{n} w_{i}^{\text {diff }} w_{i}^{\text {rank }}}, i=1, \ldots, n
$$

The PD\&R is an appealing model as it is the only model which includes the individual ranks of criteria.

\subsection{LP-GW-AHP model}

The approaches for linking the AHP and the data envelopment analysis are studied frequently. Thus we decided to include LP-GW-AHP model (Hosseinian et al., 2012), which employed concepts from the data envelopment analysis. The criteria (or alternatives) $i=1, \ldots, n$ are viewed as decision making units. Criteria (alternatives) are viewed as outputs so the model has $n$ outputs. The WGM is used for the AIJ. The group priorities $w_{1}, \ldots, w_{n}$ were derived by solving the linear programming model (12):

$\max \mathrm{z}$ subject to $w_{i} \geq \mathrm{z}, \mathrm{i}=1, \ldots, \mathrm{n}$,

$$
\begin{aligned}
& \sum_{j=1}^{n}\left(\prod_{k=1}^{m}\left(a_{i j}^{k}\right)^{\alpha_{k}}\right) v_{j}-w_{i}=0, \mathrm{i}=1, \ldots, \mathrm{n}, \\
& \sum_{i=1}^{n} w_{i}=1 \\
& v_{i}-\frac{1}{\beta} w_{i} \geq 0, \mathrm{i}=1, \ldots, \mathrm{n},
\end{aligned}
$$




$$
\begin{aligned}
& v_{i}-\frac{1}{n} w_{i} \leq 0, \mathrm{i}=1, \ldots, \mathrm{n}, \\
& w_{i} \geq 0, v_{i} \geq 0, \mathrm{i}=1, \ldots, \mathrm{n},
\end{aligned}
$$

where $\beta=\min \left\{\max _{i}\left(\frac{1}{r_{i}} \sum_{j=1}^{n} a_{i j}^{W G M M} r_{j}\right), \max _{i}\left(\frac{1}{c_{i}} \sum_{j=1}^{n} a_{i j}^{W G M M} c_{j}\right)\right\}$ and $r_{1}, \ldots, r_{n}$ and $c_{1}, \ldots, c_{n}$ are the row sums and the column sums of group comparison matrix $A^{W G M M}$, respectively.

\section{Measures for evaluating group AHP methods}

For comparisons of group AHP methods results, we need suitable measures of evaluation. We found only two existing measures for evaluating different group AHP methods in the literature. The FP index (Hosseinian et al., 2009), which is measured by the Euclidean distance:

$$
F P=\sqrt{\frac{1}{n^{2}} \sum_{i=1}^{n} \sum_{j=1}^{n}\left(a_{i j}{ }^{W G M M}-\frac{w_{i}}{w_{j}}\right)^{2}}
$$

The FP index prefers methods which employ $A^{\text {WGMM }}$ and does not shed light on the distance between the individual judgments or priorities and the final group priorities. The SAT index (Huang et al., 2009) composes differences between the priorities and differences between the ranks:

$$
\rho=\sqrt[m]{\prod_{k=1}^{m}\left(\frac{1}{n} \sum_{i=1}^{n}\left(\eta_{i}^{k}\right)^{s_{i}^{k}}\right)}
$$


where $\eta_{i}^{k}=\frac{\left(\left|w_{i}^{k}-w_{i}\right|\right)^{-1}}{\sum_{i=1}^{n}\left(\left|w_{i}^{k}-w_{i}\right|\right)^{-1}}$ and $\varsigma_{i}^{k}=\left|r_{i}^{k}-r_{i}\right|, i=1, \ldots, n$. A higher SAT index indicates greater satisfaction of DMs with the group result. The drawbacks of the SAT index are that it is not a continuous function and it is not defined if $w_{i}^{k}=w_{i}$.

We developed three new evaluation measures because of the deficiencies of the existing measures. Two measures are generalized from Euclidian distance and minimum violations from one DM’s case (Srdjevic, 2005; Mikhailov, 2006):

$$
G E D=\frac{1}{m} \sum_{k=1}^{m} \sqrt{\sum_{i=1}^{n} \sum_{j=1}^{n}\left(a_{i j}^{k}-\frac{w_{i}}{w_{j}}\right)^{2}}
$$

The group Euclidian distance (GED) measures the average sum of distances between the judgments of DMs and the related ratios of group priorities vector.

$$
G M V=\frac{1}{m} \sum_{k=1}^{m} \sum_{i=1}^{n-1} \sum_{j=i+1}^{n} I_{i j}, I_{i j}=\left\{\begin{array}{ccccc}
1 & \text { if } & w_{i}>w_{j} & \text { and } & a_{i j}<1, \\
1 & \text { if } & w_{i}<w_{j} & \text { and } & a_{i j}>1, \\
0.5 & \text { if } & w_{i}=w_{j} & \text { and } & a_{i j} \neq 1, \\
0.5 & \text { if } & w_{i} \neq w_{j} & \text { and } & a_{i j}=1, \\
0 & \text { otherwise } & &
\end{array}\right.
$$

The group minimum violations (GMV) averages the violations of each DM associated with the order reversals.

For evaluation of distances between DMs' priorities and group priorities we proposed the distance between weights (WD):

$$
W D=\frac{1}{m} \sum_{k=1}^{m} \sqrt{\sum_{i=1}^{n}\left(w_{i}^{k}-w_{i}\right)^{2}}
$$




\section{Theoretical example}

Let us consider three equally important DMs comparing four criteria and providing comparison matrices $A, B$ and $C$.

$$
A=\left[\begin{array}{llll}
1 & 5 & 3 & 1 \\
\frac{1}{5} & 1 & \frac{1}{5} & \frac{1}{7} \\
\frac{1}{3} & 5 & 1 & \frac{1}{2} \\
1 & 7 & 2 & 1
\end{array}\right], B=\left[\begin{array}{cccc}
1 & 1 & 2 & 2 \\
1 & 1 & 7 & 6 \\
\frac{1}{2} & \frac{1}{7} & 1 & 1 \\
\frac{1}{2} & \frac{1}{6} & 1 & 1
\end{array}\right], C=\left[\begin{array}{cccc}
1 & \frac{1}{2} & 2 & 7 \\
2 & 1 & 5 & 9 \\
\frac{1}{2} & \frac{1}{5} & 1 & 9 \\
\frac{1}{7} & \frac{1}{9} & \frac{1}{9} & 1
\end{array}\right]
$$

Their consistency ratios are $0.0385,0.0674$, and 0.0909 , respectively, which indicates that all comparison matrices are acceptably consistent. The group priority vectors are obtained using the WGM-AIJ, WGM-AIP, WAM, LW-AHP, GWLS, PD\&R, and the LP-GW-AHP methods. The results are presented in Table 1. The rankings of criteria for the WAM and LWAHP are identical and the priorities are similar. This similarity is due to the fact that the LWAHP is a type of WAM with unfixed weights. The rankings obtained by the other five methods are equal and the priorities of the WGM-AIJ, WGM-AIP and LP-GW-AHP are similar as they all use the geometric mean within the aggregation. The priorities of the GWLS and PD\&R method greatly differ from the priorities of other methods.

Table 2 shows the evaluation of the results from Table 1 according to the five presented evaluation measures: FP index (13), SAT index (14), group ED (15), group MV (16) and WD (17). The FP index prefers the WGM-AIJ and LP-GW-AHP, which employ the geometric mean of judgments. The SAT index indicates that the WAM and LW-AHP are the best methods. Group ED favors the LW-AHP method. Group MV is equal for all group AHP methods. The WD prefers the LW-AHP and WAM. The results showed that in our example the LW-AHP was the best group AHP method according to most criteria. The methods WGM-AIJ, WGM-AIP, WAM and LP-GW-AHP had some advantages and weaknesses and 
their evaluation measures placed them in the middle of the rankings. The priorities of the GWLS and PD\&R methods were outstanding and the evaluation measures confirmed that other methods provided more suitable group priority vectors.

\section{Case study}

A study was conducted to select the most suitable construction type among solid wood construction, wood-frame construction, aerated concrete, brick construction, and steel construction, for industrial buildings. The construction of prefabricated industrial wooden buildings today is supported by strong arguments. Innovations and improvements introduced in the early 1980s have helped to promote wooden prefabricated buildings around the world. Further, in construction of industrial buildings, the following adjustments are very important: (a) the transition from on-site construction to industrial prefabrication, (b) the transition from stick-building to modular construction, (c) an increased use of glued lumber in construction, (d) the development of environmentally friendly solutions for wood protection, and (e) the shift from small to large panel system (LPS) construction (Žegarac \& Leskovar, 2012). The choice of the material is the most important decision and it has long-term consequences for the owner of the structure (Johnson, 1990).

The goal of the case study was to select the most suitable construction type for industrial buildings. According to the criteria different construction materials for industrial buildings were compared: solid wood construction, wood-frame construction, aerated concrete, brick construction, and steel construction. The research was carried out by survey, which included experts from several European countries, such as architect engineers, construction engineers, and wood-technology engineers. The selection of the criteria was conducted by the Delphi method (Gupta \& Clarke, 1996). In the first round of the decision making process experts selected eighteen most important construction criteria and grouped them into five categories. 
In the second round nine most important criteria out of the eighteen were selected. They were classified into three groups: (1) Mechanical and technical criteria (energy efficiency, load capacity, form and dimension planning limitations-FDP limitations), fire safety), (2) Economic criteria (construction costs, depreciation costs, construction time), and (3) Residential criteria (aesthetics, quality of living). These nine criteria were included in the AHP decision tree (Fig. 1). The pairwise comparisons of criteria were conducted by twentyseven experts. The highly inconsistent comparison matrices with $C R>0.18$ were rejected. The most inconsistent judgments in comparison matrices with $0.1<C R<0.18$ were adjusted. In the end, fifteen accepted comparison matrices had $C R<0.1$.

Three groups of experts consisting of five wood-technology engineers, four architect engineers, and six construction engineers, with different respects to the constructing criteria, took part in the research. Since more homogenous judgments within the groups than between the groups were expected, we suggested two new methods: the WGM-WAM and WGM-LWAHP, which were used for the AIJ within each group of engineers and for the AIP of the three expert groups. In both methods we used the WGM-AIJ for deriving the priority vectors of each group of experts. In the WGM-WAM method, the WAM was applied and in the WGMLW-AHP method, the LW-AHP was applied for aggregating three group vectors into the final priority vector. The results of all eight group methods are presented in Table 3 . All methods put fire safety, load capacity and energy efficiency first, and aesthetics last. Despite that, the PD\&R method stands out with high priorities.

An evaluation of all nine methods with the same five measures used in the theoretical example was conducted. The results are presented in Table 4. The measures evaluated the methods very heterogeneously. This indicated that no group AHP method is "the best". 
Nevertheless, the methods with the highest evaluations according to most of the measures were WGM-WAM and WGM-LW-AHP and they evaluated better than the unmodified WAM and LW-AHP methods, respectively. This signified that it was useful to create smaller homogenous groups of DMs. The evaluation of the PD\&R method was the least favorable, followed by the GWLS method.

Five different types of construction materials were assessed separately for each of the nine key criteria of construction. The weighting coefficients of the construction costs criterion were selected on the basis of average costs per square meter of selected wall types by chosen manufacturers. Depreciation costs were assessed in relation to the service life of the material and construction costs. The criterion of form and dimension planning limitations was estimated on the basis of indicators such as functionality, span possibility, multistory construction, system solutions and surface efficiency. Factors such as prefabrication level, drying, transport, knowledge and experience in using the elements affected the estimation of the construction time criterion. The quality of living was assessed on the basis of health and psychological factors. The weighting coefficients for the aesthetics criterion were selected on the basis of the survey. For three out of nine criteria (load capacity, fire safety, and energy efficiency) the parameters were set as the construction standards. With regard to the fact that with proper planning requirements of limited standards for each of these materials can be met, all construction types were ascribed the same weighting coefficients for the criteria of load capacity, fire safety, and energy efficiency. The results are presented in Table 5.

The final priorities for the types of construction given in Table 6 were calculated as the weighted sums of priorities from Table 5, where the weights in the weighted sums are the priorities from Table 3. The results of all group methods, except PD\&R, indicated that wood 
frame construction was the most appropriate for industrial building. Concrete construction was placed second, followed by solid wood, steel frame and brick construction as the last. Let us comment that the differences between the priorities of different types of construction are small. So even though brick construction was ranked last, it did not mean that brick construction is completely inappropriate for industrial building.

The findings of the study serve as a starting point for investors, civil engineers, architects and the others involved in building process to understand the importance of the selection criteria, however, further studies on a larger scale are needed to confirm these observations. On the methodological part, the group results were compared with the five evaluation measures GED, GMV, WD, SAT index, FP index. The results of the evaluation showed that in the case of a non-homogenous group of decision makers, dividing the group into smaller, homogenous groups and applying the newly developed WGM-WAM or WGM-LW-AHP methods produced better results. We do not recommend the PD\&R and GWLS methods until their regularity is more thoroughly investigated.

\section{Conclusions and further work}

In order to evaluate group AHP methods we developed three new measures: group Euclidean distance, group minimum violations, and distance between weights for evaluating group AHP methods. We applied these new measures in addition to the existing SAT index and FP index measures in a theoretical example and in a case study of industrial building construction. The aim of the theoretical example was evaluation of seven carefully selected AHP group methods techniques, which are appealing for use in many applications. Our comparison indicated that the LW-AHP model performed the best of the selected AHP group methods. Therefore, we recommend applying the LW-AHP model to real-world group decision making scenarios. 
The goal of the case study was to select the most suitable construction material for industrial buildings based on nine mechanical, technical, economic, and residential criteria. Three groups of engineers took part in the survey. We presumed that judgments will be more homogenous within each group of engineers than between groups. We proposed two new two stage group approaches, WGM-WAM and WGM-LW-AHP to aggregate individual judgments into group priority. We applied seven group AHP methods from the theoretical example and two new approaches to evaluate five different types of construction materials in the case study. The results showed that wood frame construction was the most appropriate for industrial buildings. The advantages of wood as a construction material with lower embodied global warming potential, and embodied carbon positively associated with well-being, aesthetic and eco-friendliness, and realistic end-of-life disposal options (Praznik et al., 2014).

The AHP group methods have been only evaluated in two examples, which is the main limitation of our study. Therefore some open issues remain, which should be studied in the future. For each method its robustness and the stability of the solution should be studied. In addition, theoretical analysis of evaluation measures should be investigated and the correlation between them examined. The results of evaluation depend on the definition of the evaluation measure. The best result was achieved by the method that minimizes this measure. In this way the evaluation measure can become a group method.

\section{Acknowledgement}

Manja Kuzman would like to acknowledge the Slovenian Research Agency for financial support within the frame of the program P4-0015 and Ministry of Education, Science and Sport RS in the frame of the WoodWisdom-Net+ project W3B Wood Believe. 


\section{References}

Ahmad, F., Saman, M.Y.M., Mohamad, F.S., Mohamad, Z., \& Awang, W.S.W. (2014). Group Decision Support System Based on Enhanced AHP for Tender Evaluation. International Journal of Digital Information and Wireless Communications (IJDIWC), 4(2), 248-257.

Ananda, J., \& Herath, G. (2008). Multi-attribute preference modelling and regional land-use planning. Ecological Economics, 65, 325-335.

Bryson, N., \& Joseph, A. (1999). Generating consensus priority point vectors: a logarithmic goal programming approach. Computers \& Operations Research, 26, 637-643.

Cho, Y.G., \& Cho, K.T. (2008). A loss function approach to group preference aggregation in the AHP. Computers \& Operations Research, 35, 884-892.

Cortés-Aldana, F.A., García-Melón, M., Fernández-de-Lucio, I., Aragonés-Beltrán, P., \& Poveda-Bautista, R. (2009). University objectives and socioeconomic results: A multicriteria measuring of alignment. European Journal of Operational Research, 199, 811-822.

De Brucker, K., Macharis, C., \& Verbeke, A. (2013). Multi-criteria analysis and the resolution of sustainable development dilemmas: A stakeholder management approach. European Journal of Operational Research, 224(1), 122-131. 
de Luca, S. (2014) Public engagement in strategic transportation planning: An analytic hierarchy process based approach. Transport Policy, 33,110-124.

Dyer, R.F., \& Forman, E.H. (1992). Group decision support with the Analytic Hierarchy Process. Decision Support Systems, 8, 99-124.

Escobar, M.T., \& Moreno-Jimenez, J.M. (2007). Aggregation of individual preference structures in AHP-group decision making. Group Decision and Negotiation, 16, 287-301.

Forman, E., Peniwati, K. (1998). Aggregating individual judgments and priorities with the analytic hierarchy process. European Journal of Operational Research, 108, 165-169.

Hämäläinen, R., \& Pöyhönen, M. (1996). On-line group decision support by preference programming in traffic planning. Group Decision and Negotiation, 5, 485-500.

Hosseinian, S., Navidi, H., \& Hajfathaliha, A. (2012). A new linear programming method for weights generation and group decision making in the analytic hierarchy process. Group Decision and Negotiation, 21, 233-254.

Huang, Y.S., Liao, J.T., \& Lin, Z.L. (2009). A study on aggregation of group decisions. Systems Research and Behavioral Science, 26, 445-454.

Ishizaka, A., \& Labib, A. (2011a). Selection of new production facilities with the Group Analytic Hierarchy Process Ordering method. Expert Systems with Applications, 38(6), 7317-7325. 
Ishizaka, A., \& Labib, A. (2011b). Review of the main developments in the analytic hierarchy process. Expert Systems with Applications, 38(11), 14336-14345.

Kuzman, M.K., Grošelj, P., Ayrilmis, N., \& Zbasnik-Senegacnik, M. (2013). Comparison of passive house construction types using analytic hierarchy process. Energy and Buildings, 64, 258-263.

Lai, V.S., Wong, B.K., \& Cheung, W. (2002). Group decision making in a multiple criteria environment: A case using the AHP in software selection. European Journal of Operational Research, 137, 134-144.

Lee, A.H.I., Chang, H.J., \& Lin, C.Y. (2009). An evaluation model of buyer-supplier relationships in high-tech industry - The case of an electronic components manufacturer in Taiwan. Computers \& Industrial Engineering, 57, 1417-1430.

Lipovetsky, S. (2009). Global priority estimation in multiperson decision making. Journal of Optimization Theory and Applications, 140, 77-91.

Mikhailov, L. (2004). Group prioritization in the AHP by fuzzy preference programming method. Computers Operations Research, 31, 293-301.

Moreno-Jimenez, J.M., Aguaron, J., \& Escobar, M.T. (2008). The core of consistency in AHP-group decision making. Group Decision and Negotiation, 17, 249-265. 
Peniwati, K. (2007). Criteria for evaluating group decision-making methods. Mathematical and Computer Modelling, 46, 935-947.

Praznik, M., Butala, V., \& Zbašnik - Senegačnik, M. (2014). A simple method for evaluating the sustainable design of energy efficient family houses. Strojniški vestnik, 60(6), 425436.

Regan, H.M., Colyvan, M., \& Markovchick-Nicholls, L. (2006). A formal model for consensus and negotiation in environmental management. Journal of Environmental Management, 80, 167-176.

Ren, J., Fedele, A., Mason, M., Manzardo, A., \& Scipioni, A. (2013). Fuzzy Multi-actor Multi-criteria Decision Making for sustainability assessment of biomass-based technologies for hydrogen production. International Journal of Hydrogen Energy, 38(22), 9111-9120.

Saaty, T.L. (1980). The Analytic Hierarchy Process. McGraw-Hill: New York.

Saaty, T.L, \& Vargas, L.G (2007) Dispersion of group judgments. Mathematical and Computer Modelling, 46(7-8), 918-925.

Skorupski, J. (2014) Multi-criteria group decision making under uncertainty with application to air traffic safety. Expert Systems with Applications, 41(16), 7406-7414.

Srdjevic, B. (2007). Linking analytic hierarchy process and social choice methods to support group decision-making in water management. Decision Support Systems, 42, 2261-2273. 
Srdjevic, Z., Lakicevic, M., \& Srdjevic, B. (2013). Approach of Decision Making Based on the Analytic Hierarchy Process for Urban Landscape Management. Environmental Management, 51(3), 777-785.

Sun, L., \& Greenberg, B. (2006). Multicriteria Group Decision Making: Optimal priority synthesis from pairwise comparisons. Journal of Optimization Theory and Applications, 130, 317-339.

Sun, J., Li, H. (2009). Financial distress early warning based on group decision making. Computers \& Operations Research, 36, 885-906.

Taylor, A.D., \& Pacelli, A.M. (2008). Mathematics and Politics: Strategy, Voting, Power and Proof. second edn. Springer Verlag: New York.

Van den, R.C. \& Honert, R.C. (1998). Stochastic group preference modelling in the multiplicative AHP: A model of group consensus. European Journal of Operational Research 110: 99-111.

Wang J.Q, Peng, L., Zhang, H.Y. \& Chen, X.H. (2014). Method of multi-criteria group decision-making based on cloud aggregation operators with linguistic information. Information Sciences, 274, 177-191.

Yu, L., Lai, K.K. (2011). A distance-based group decision-making methodology for multiperson multi-criteria emergency decision support. Decision Support Systems, 51(2), 307315. 
Leskovar, V.Z., \& Miroslav, P. (2012). Influence of glazing size on energy efficiency of timber-frame buildings. Construction \& building materials, 30, 92-99.

\section{Figure caption}

Figure 1. The AHP decision tree with criteria and alternatives in the construction of industrial buildings

\section{Table list}

Table 1: The group priorities and the ranking (r) of criteria, according to the WGM-AIJ, WGM-AIP, WAM, LW-AHP, GWLS, PD\&R, and LP-GW-AHP model

Table 2: The results of the evaluation of group priorities according to the FP index, SAT index, GED, GMV and WD and the rankings of the group AHP methods

Table 3: The group priorities and the rankings (r) of construction criteria, according to the WGM-AIJ, WGM-AIP, WAM, WGM-WAM, LW-AHP, WGM-LW-AHP, GWLS, PD\&R and the LP-GW-AHP models

Table 4: The results of the evaluation of group priorities of construction criteria according to the FP index, SAT index, GED, GMV and WD and the rankings of the group AHP methods Table 5: The priorities of alternatives for construction of industrial buildings regarding the construction criteria

Table 6: The final priorities and ranks (r) of different types of construction for industrial buildings 